\title{
The Alberta Oil Sands Tailings Roadmap Project
}

\author{
J.C. Sobkowicz Thurber Engineering Ltd., Canada
}

J.E.S. Boswell Thurber Engineering Ltd., Canada

M.D. Kofoed Thurber Engineering Ltd., Canada

I.D.C. Gidley Thurber Engineering Ltd., Canada

\begin{abstract}
In 2011, Alberta Innovates - Energy and Environment Solutions (AI-EES), Canada awarded a contract to the Consortium of Tailings Management Consultants (CTMC) to prepare an Oil Sands Tailings Technology Deployment Roadmap. In this paper the work done in terms of the contract is referred to as the project. The project objective was to create a roadmap outlining further research and development needs for promising tailings treatment / dewatering technologies.
\end{abstract}

This paper describes the objectives and main components of the project, the technical process whereby these goals were met, and the key recommendations arising from the project work.

Recognising that the development of tailings roadmaps may hold potential for mining sectors other than the Canadian oil sands, the authors reflect on some of the key observations and conclusions from the study, and offer some insights and recommendations about the technology assessment and roadmap development process that was employed.

\section{Introduction}

Traditional oil sands mines in the Fort McMurray region of Alberta, Canada produce large amounts of both solid and fluid tailings. Mining and tailings production have been ongoing over the past 40 years, and are expected to continue with increased production rates for decades to come. Oil sands operations are subject to continuing concerns such as geotechnical and environmental risks, as well as the exponentially growing long-term liability associated with increased tailings production, and the reclamation burden it involves.

In response to growing concerns within the industry, in early 2011 Alberta Innovates - Energy and Environment Solutions (AI-EES), in partnership with the Oil Sands Tailings Consortium (OSTC), awarded a contract to the newly formed Consortium of Tailings Management Consultants (CTMC) to proceed with a unique project titled, "The Technology Deployment Roadmap and Action Plan for 'End-to-End' Solutions for Oil Sands Tailings". The project objective was:

"... to create a technology deployment roadmap and action plan that will assist regulators and industry to create and implement technology solutions that will meet the goals of Alberta Environment's (AE's) Draft Tailings Management Framework and the Energy Resources Conservation Board (ERCB) Directive 074" (Alberta Innovates, 2010).

The Technology Deployment Roadmap project was a joint initiative on the part of government and industry to cultivate and support a broader strategy for continuing sustainable management of tailings produced in the oil sands.

The project had widespread involvement, incorporating input from government organisations and departments including:

- Alberta Innovates - Energy and Environment Solutions

- Alberta Energy 
- Alberta Environment and Sustainable Resource Development

- Energy Resources Conservation Board

- CANMET

Member companies of the OSTC that took part in the project were:

- Canadian Natural Resources Limited

- Imperial Oil

- Shell Canada Energy

- Suncor Energy Inc.

- Syncrude Canada

- Teck Resources Limited

- Total E\&P Canada Ltd

The OSTC and government organisations were responsible for driving, guiding and reviewing the project's progress; the consortium of engineering firms was tasked with the development of the project as a whole. The assembled team thus included tailings management consultants and academics. This group was referred to as the CTMC, and included:

- AMEC

- BGC Engineering

- Golder Associates Ltd

- Klohn Crippen Berger Ltd

- Norwest Corporation

- Thurber Engineering Ltd

- The University of Alberta Geotechnical Group

Upon commencement of the project, an execution plan was produced by the CTMC to define the project goals, namely that the final product was to consist of a framework that will allow government and industry to:

1. Help achieve more timely deployment of end-to-end tailings technologies, and share the results and knowledge of tailings deployment activities.

2. Document the current state of tailings reclamation technology and define technology pathways to reach a fully reclaimed landscape.

3. Serve as a basis for accessing government and industry funding to accelerate commercial-scale demonstration of technology, and to promote comprehensive information sharing and technology transfer.

4. Identify technology options and establish a framework for oil sands operators to conduct their own detailed feasibility studies and deploy technology, and allow regulators to verify performance during this process.

5. Promote a collaborative approach to oil sands tailings technology that expedites technology deployment, reduces environmental impacts beyond the boundaries of the mine lease, and enhances public trust.

6. Provide a medium for sharing the results and knowledge of effective tailings deployment initiatives. 
The final report released to the public consisted of five separate volumes; each volume pertains to a unique project segment. Volume 1 summarised the results of the project, while Volumes 2 through 5 are reports from the individual project teams; these volumes provide an in-depth perspective of the work completed, and the current status of the oil sands tailings industry (Consortium of Tailings Management Consultants, 2012a, b, c, d, e).

This was a complex project that covered 18 months of work and produced five large reports. The reader is encouraged to examine the project report volumes online at http://ai-ees.ca/reports.aspx or http://www.cosia.ca/projects/tailings/tailings-technology-roadmap.

For some background on the various types of oil sand tailings and the unique tailings terminology used in this industry, the reader is referred to Sobkowicz and Morgenstern (2009).

\section{Methodology}

The scope of the project was divided into four separate components. Each component had a team lead (and alternate) who were assisted by project support staff and technical specialists. The scope of each component is as follows:

Component 1 (C1) was to search, collect and compile available information on the current status of any technology with some potential applicability to oil sands mines. The information collected was synthesised into a summary of the current industry standard tailings management practices, and used to establish a comprehensive database of technologies. All technologies had a standardised technology information sheet filled out for them to ensure ease of reference. BGC Engineering and Norwest Corporation completed the C1 work jointly.

Component 2 (C2) was to develop a set of tailings management and reclamation objectives. The objectives were used to develop criteria used to evaluate the technologies listed by the C1 team. Klohn Crippen Berger completed the $\mathrm{C} 2$ work.

Component 3 (C3) was responsible for setting up and completing a review of the selected technologies against the tailings management and reclamation objectives determined by $\mathrm{C} 2$. The evaluation output was to be used to identify gaps, strengths and improvement opportunities within the current technologies and technology suites. Golder Associates Ltd. and the University of Alberta carried out the C3 work.

Component 4 (C4) was responsible for the identification of 'highlighted' technologies that could improve existing tailings treatment suites, or had the potential to contribute to idealised new suites. Upon identification of these technologies, C4 was also to develop tailings technology deployment roadmaps for the technologies (referred to simply as tailings roadmaps herein). The roadmaps consisted of an outline of certain items that required consideration for the technologies to be able to progress through research and development stages to commercial application in an oil sands mine. Thurber Engineering Ltd. and AMEC carried out this work jointly.

To ensure continuity throughout the project, the work was carried out under the guidance of a Research Advisory Committee, which consisted of representatives from government, industry and the CTMC. During each stage of the project, client review workshops and Research Advisory Committee meetings were held to review and discuss progress, and to provide input as to the direction of future work. The workshops provided ample time to review process efficiency, and determine how the completed work would contribute to the project goals.

\section{Data and results}

\subsection{Technology discovery and formation of the database}

Through the course of their work, C1 identified 549 technologies (from mining through to reclamation) with the potential to improve oil sand tailings practises. Review of information reduced the list to 101 unique technologies, not including variations and enhancements. Technologies were categorised by their stage in 
the mining life cycle, corresponding to one of the following groups: mining, extraction, tailings processing, tailings capping/deposition, water treatment, and reclamation. Technologies were labelled by their stage in the research and development cycle (research, development, or commercially implemented); these labels were specific to a technology's use or potential applicability in the oil sands.

As part of their work, the $\mathrm{C} 1$ team produced a comprehensive description of current mining, extraction and tailings treatment/deposition practices in the oil sands industry. This led to the definition of eight separate mining/extraction/tailings technology suites. These suites were chosen to emphasise the 'end-to-end' technology sets used, which were capable of following the entire process from the start of mining to the end of reclamation. The suites are:

- Conventional hydraulic fill.

- Water-capped Mature Fine Tailings (MFT).

- Composite tailings (CT) and non-segregating tailings (NST).

- Conventional tailings thickening.

- In-line thickening of tailings, with thin lift deposition/dewatering.

- In-line thickening of tailings, with thick lift deposition/accelerated dewatering.

- Centrifuged MFT.

- Coke capping.

Twenty-two separate, unique technologies are already commercially used in the oil sands. Many of these technologies are mature and well known, while others are the result of recent industry development undertaken in response to an established concern.

\subsection{Benchmarking the process}

To proceed with an analysis of the technologies identified by $\mathrm{C} 1$, the $\mathrm{C} 2$ team developed a series of tailings management and reclamation objectives. These were confirmed with the OSTC and government regulators. The objectives were aligned to respect the desires of a broad range of stakeholders, including mining companies and investors, first nation communities, non-governmental organisations, local communities, consumers, media, and various government departments.

The main tailings reclamation and management objectives are:

1. To minimise production and long-term storage of fluid-fine tailings.

2. To manage tailings in a manner that minimises the impact of process-affected water on the environment.

3. To facilitate progressive reclamation and achieve a trafficable surface as soon as possible, following the cessation of deposition.

4. To reduce ongoing operations liability and long-term closure liability.

5. To minimise the footprint of permanent tailings facilities.

6. To minimise the cost of construction, operations, and reclamation without compromising safety.

7. To use robust technologies.

8. To enhance the potential of technologies to reach commercial implementation.

\subsection{Standard evaluation of technologies}

The C3 technology evaluation process was carried out in a series of workshops. The assessments were completed by experts from the oil sands industry, selected based on their knowledge of the different 
aspects of the mining, process and tailings life cycle. The output of these evaluations was considered representative of industry opinion. Although the process was not without flaws, it reflected the best judgement of the team of evaluators at the time of the workshops.

The results were used to define the strengths and weaknesses of each technology, and the ways that each could be modified or improved, to better achieve the tailings reclamation and management objectives. This information was to be incorporated into the $\mathrm{C} 4$ work in order to develop a better understanding of how each technology fit into a tailings roadmap, or how it could be implemented as a gap filling technology in another tailings technology suite.

\subsection{Technology prioritisation}

The $\mathrm{C} 4$ team was tasked with producing the tailings roadmaps. Information from the previous component reports was consolidated and reviewed thoroughly to determine the validity of individual technologies, as well as their development status.

The initial $\mathrm{C} 4$ work focused on identifying technologies from the $\mathrm{C} 1$ database that had a high potential to improve any of the existing commercial tailings suites or to contribute to a new technology suite. Technologies in the research or development stages were assembled into a list of 'highlighted' technologies that required further development and were the focus of further consideration. Highlighted technologies are listed in Table 1.

\section{Table 1 Highlighted technologies}

\begin{tabular}{ll}
\hline Technology\# & Description \\
\hline T-606 & Mobile in-pit crusher \\
T-600 & Fine sizing (crushing) \\
T-602 & Secondary (deep cone) separation vessel \\
T-604 & High temperature heating (for solvent removal) \\
T-607 & Mobile versions of conventional separation extraction equipment \\
T-024 & Alberta Taciuk process \\
T-186 & Solvent extraction \\
T-548 & Retort-based bitumen extraction \\
T-603 & Control of biogenic gas during mature fine tailings spiking \\
T-040 & Thin lift drying Robinsky cones \\
T-032 & Accelerated dewatering \\
T-037 & Thin layer freeze-thaw cycling dewatering (T-037) \\
T-039 & Accelerated evapotranspiration using vegetation \\
T-062 & (Variation) co-mixing overburden and centrifuge cake \\
T-065 & Interlayered centrifuge cake and sand - variation of interlayer mature fine \\
& tailings and sand \\
T-088 & Shock densification \\
T-090 & Vertical drains \\
T-099 & Stacker hydro-cyclones (including fully mobile versions) \\
T-188 & Under-drained tailings \\
\hline
\end{tabular}




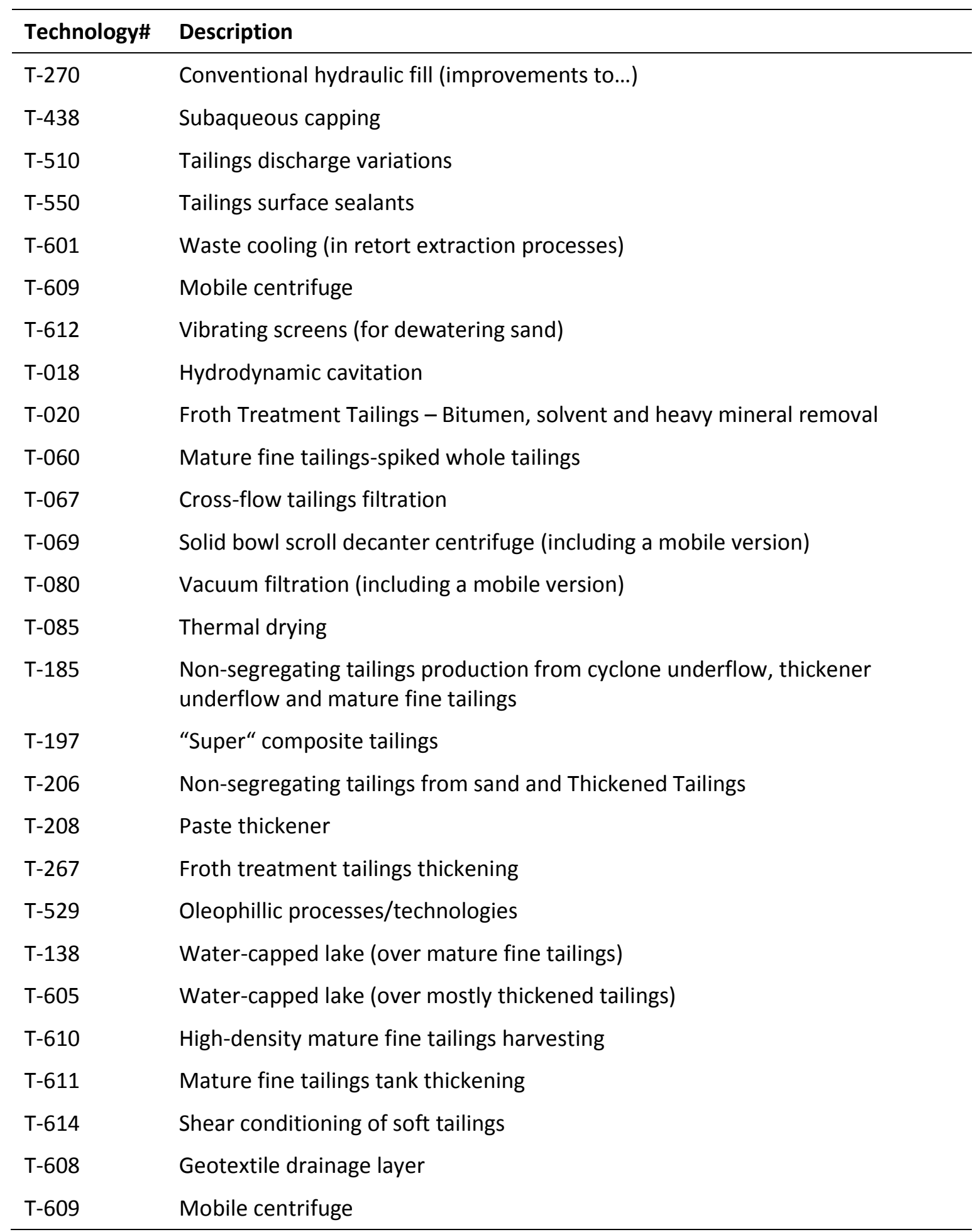

The final stage of the C4 evaluation was to determine the benefits, risks and costs of the specified technologies, and these were illustrated on several charts, an example of which is shown in Figure 1. 


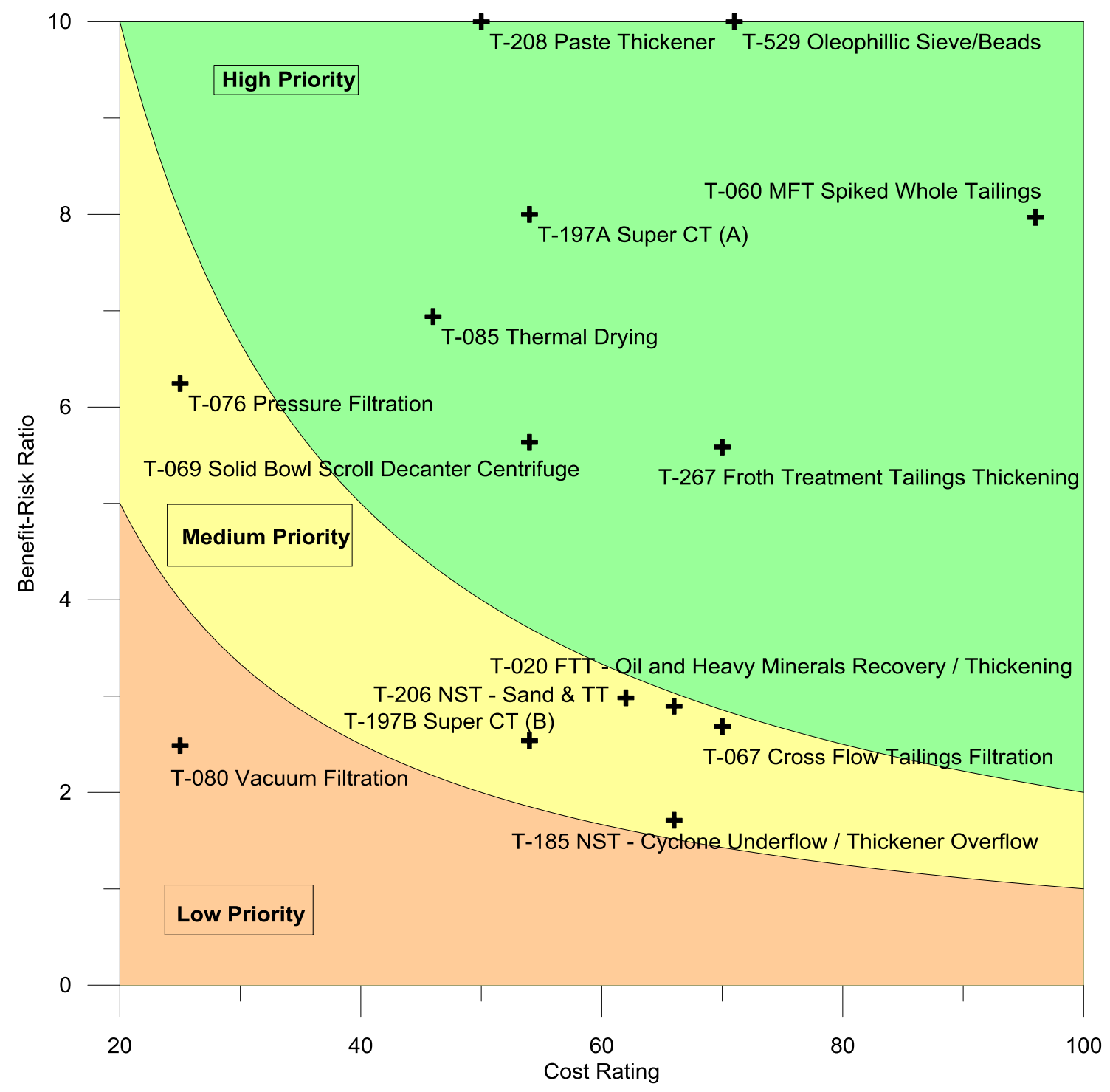

\section{Figure 1 Tailings processing technology prioritisation}

\subsection{The technology deployment roadmap}

The ultimate product of the project was to produce several tailings roadmaps that would focus on different aspects of the current state of the industry. Each roadmap centred on a separate theme or application, thereby covering multiple technologies in the research or development state.

Complimentary to the review of current technologies, the $\mathrm{C} 4$ team developed a generic model of oil sands technology development to provide a base reference for the roadmaps. The purpose of the model was to visualise the development of a technology and chart a course for the technology to follow through development to commercial implementation. The generic technology development model was vital to the systematic evaluation and positioning of technologies in the development spectrum. The conception and structure of this model is discussed in a separate paper presented at these proceedings (Gidley and Boswell, 2013). 


\section{Table 2 Technology development roadmaps were completed for these suites}

\begin{tabular}{cl}
\hline Roadmap no. & Related tailings technology suite name \\
\hline 1 & Centrifuging MFT with conveyor/stacking \\
2 & Composite tailings \\
3 & In-line thickening with accelerated dewatering \\
4 & In-line thickening with thin lift evaporative drying \\
5 & Thickening \\
6 & Water capped end pit lake \\
7 & Improvement to water-based extraction \\
8 & Non-aqueous solvent extraction. Retort based extraction. Parallel high/low fines suite \\
9 & In-pit tailings stream \\
\hline
\end{tabular}

Each roadmap was created by: (a) defining all of the components of a selected technology suite; (b) identifying components with the potential for improvement; and (c) identifying technologies that could enhance and benefit the technology suite through further development. Each roadmap was structured as follows:

1. Suite introduction - a short description of the purpose and the process mechanics of the technology suite.

2. Improvement opportunities - a table summarising the improvement opportunities and respective gap filler technologies identified during the $\mathrm{C} 4$ work.

3. Technology breakdown - categorisation of the identified gap filler technologies/suite components by their developmental status (research, development, commercial or new). Only research and development technologies were further analysed.

4. Suite diagram - sketches/diagrams displaying the technology process for commercial suites, or proposed flow charts for new suggested suites. A sample schematic follows this list.

5. Suite specific R\&D model - the generic research and development model developed by the C4 team was populated with the individual technologies specific for each identified technology suite. The location of a technology on the graphic was used to reflect the technology's current stage of development.

6. Technology deployment table - a comprehensive look at individual technologies and their capabilities.

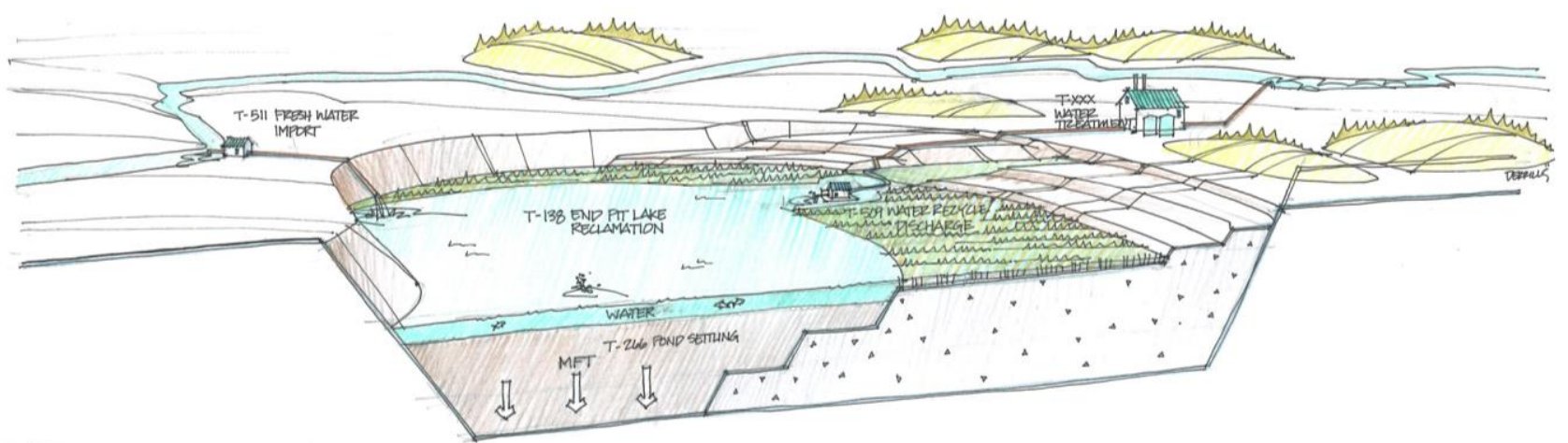

Figure 2 Water-capped end pit lake technology suite 
Emphasis was on the technology suites that would be brought into the roadmap process. However, the substance of the roadmaps was in describing the further research and development needed to bring these technologies to full commercial implementation. A technology deployment table was completed in every roadmap for each identified technology in the research or development stage. Each table was broken down to present and expand upon specific information:

1. Technology name.

2. Technology number - the number assigned by $\mathrm{C} 1$ and used for reference.

3. Parent technology suite - identification of the technology suite(s) that the technology is a component of.

4. Potential technology contribution - identification of the technology capability, either as a gap filler to a current suite, component of a current suite or a promising technology.

5. Technology priority status - the priority assigned as a result of the $\mathrm{C} 4$ process.

6. Background information - information sheets outlining the costs, risks and benefits.

7. Development status - the current development status of the technology, supported by previous analogues from academia and industry.

8. Expected performance - expected performance and results of using the technology.

9. Steps to advance the technology - steps required to advance the technology development, comprised of generic and specific steps for each technology.

10. Expected timeline - an identification of the expected timeframe to advance the technology through the next few stages of technology development.

11. Barriers and roadblocks to success - issues that may prevent successful development and implementation of the technology.

12. Remedies and measures to overcome these barriers - identification of potential solutions to address the identified roadblocks and drive further development.

The similar structure of each roadmap enabled easy reference, and could be used to display similarities or differences between the various technology suites in a manageable fashion.

The roadmaps identify and provide information on the current status of individual technology suites. They provide information on what technologies need to make further progress, and they discuss some of the steps needed to get there.

The roadmaps also identify technological constraints and opportunities. They thus can be used to set the course for short-term future development, while at the same time planning for the long-term, and provide guidance through the development path.

\section{Conclusions}

The main conclusions from the tailings technology roadmap project are listed below. A more comprehensive, detailed list of conclusions can be found in the project report.

1. There is still no 'silver bullet' tailings technology, i.e. a single technology or suite of technologies, which will solve all the oil sands tailings challenges with a single effort.

2. A set of 9 Tailings Technology Deployment Roadmaps has been developed by the project. The roadmaps consider improvements to existing technology suites and contributions to potentially new suites, and identify technologies with a high potential to effect these changes.

a. Many different improvement opportunities have been identified across a number of technology suites. 
b. A significant number of technologies have been highlighted to address these opportunities, and represent substantial potential for further development in the oil sands.

c. The development of individual technologies has been plotted on a detailed generic model graphic to map out the steps required in their future research and development.

3. There is a major opportunity to increase the performance and decrease the cost of existing commercial tailings technologies and suites employed at the oil sands operations. Some of the main identified opportunities include:

a. Reducing segregation during beaching (for conventional tailings, composite tailings and thickened tailings). Potential improvement technologies are mostly centred on increasing the density of the tailings stream - by thickening the fines stream (directly, or as a technology component; various types of tank or in-line thickeners) or by removing water from the sand stream (through hydrocycloning or filtration). For these, chemical amendments and techniques that remove bitumen from the tailings streams have potential as enablers. Other technologies focus on reducing the amount of shear during deposition (e.g. tremie diffuser).

b. Increasing deposit strength and improving trafficability, or alternatively, capping very soft deposits (for conventional tailings, composite tailings, thickened tailings, accelerated dewatering and centrifuging mature fine tailings). Potential improvements focus on: (i) removing water through drainage (e.g. vertical drains or horizontal drainage layers); (ii) drying (using thin-lift deposition) or freeze-thaw; (iii) chemical amendments to increase strength; or (iv) soft soil capping technologies (hydraulic or mechanical).

c. Improving fines capture/storage (for conventional tailings and composite tailings). Potential improvement technologies focus on introducing additional fines into the tailings stream, either by fines spiking or by using a denser fines stream as a technology component (e.g. for composite tailings, making it with highly thickened fines).

d. Addressing geotechnical risks (for conventional tailings and composite tailings). Potential improvement technologies focus on densifying the material to increase strength or decrease the risk of liquefaction (using technologies that promote drainage or increase density before discharge).

e. Reducing long-term settlement (for thickened tailings, accelerated dewatering and centrifuged mature fine tailings). Potential technologies focus on improving the initial density of the tailings stream (through better thickening, which generally relies on new chemical amendments), or speeding up consolidation through enhanced drainage (e.g. vertical drains).

f. Addressing long-term environmental impacts (mentioned for conventional tailings and composite tailings, but likely applicable to most of the commercial technology suites). Potential improvement technologies focus on controlling release water chemistry (which might be addressed by the appropriate selection of chemical amendment technology or by water treatment), or recovering bitumen and/or heavy metals from the tailings streams.

g. For technologies that rely partially on drying to remove water from tailings streams (in-line flocculation with thin-lift dewatering, accelerated dewatering, and centrifuging of mature fine tailings), there are specific improvements that are needed, such as reducing drying area requirements and improving the consistency of the processed tailings stream. Not all of these issues are applicable to all of the technology suites mentioned, but potential technologies focus on improved thickening (usually, with better chemical amendments), pre-treating the initial tailings stream to remove bitumen (e.g. using an oleophillic sieve or similar technology), modifying discharge methods (e.g. use of central-point discharge), or enhancing the effects of drying with freeze-thaw techniques. 
h. Separating fines rich from fines poor ores and then treating the two ore streams separately. Potential improvement technologies include selective mining and the use of non-water-based extraction technologies.

i. Keeping tailings streams separated for processing and deposition if they are separated for whatever reason, (e.g. using a cyclone). This is already done in producing thickened tailings, but there may be other improvement opportunities of this nature.

j. The water-capped fluid fine tailings technology suite has its own specific improvement opportunities, many of which are related to achieving a higher density of the tailings being capped. For example, potential technologies include centrifuging or thickening (in-line or tank) of mature fine tailings, or high-density mature fine tailings harvesting from existing ponds.

4. The typical time frame required to progress a technology through the research stage is from two to three years, and through the development stage from two to five years. Additional time may be required after initial commercial implementation to bring the technology to a mature state - in the order of from one to five or more years. Timelines for bringing potential tailings technologies to full commercial implementation are thus quite long (from five to more than 10 years); this fact must be recognised when planning tailings management improvements (to reach desired reclamation and closure objectives, or in responding to regulatory changes).

\subsection{Main recommendations}

The work of all parties associated with this project demonstrates the very important benefits of full cooperation and communication amongst operators, as well as collaboration between operators, regulators, and consultants. The efforts to date are to be lauded, but this is not the time to relax focus on solving the very real tailings issues. Rather, it is a time when this past success should be leveraged, and for this reason, the following major recommendations were made by the project:

1. As a follow-up to the Tailings Technology Deployment Roadmaps included herein, it is suggested that the following steps be taken:

a. The Roadmaps should be assessed by individual operators for applicability to their specific site(s), mining practices, and tailings inventories, so that company priorities for research and development can be set.

b. More detailed research and development plans should be generated from the selected Roadmaps and specific tailings technologies. There is a role for both operators and regulators to play in this process (further discussed below).

2. In regards to the previous point, the Roadmaps and related information should be regularly revised, so that they do not become dated. This should include:

a. Setting up a process to receive additional tailings technology as it becomes available.

b. Soliciting information from credible and experienced vendors who have so far not become involved in oil sands tailings.

c. Lowering the threshold for potential vendors to participate in the Roadmap process. This should include defining what 'entrance' information is needed and standards for data submitted to the government and operators (including such items as protocols for sampling and laboratory testing).

d. As the technology database is updated, the technology evaluations and Roadmaps should also be updated to reflect the new information.

3. The Roadmaps given in the report have focused largely on Tailings Processing and Tailings Deposition \& Capping technologies. More focused study and attention should be given to 
potential technologies from the other mining life-cycle categories (Mining, Extraction and Reclamation) that could contribute to better tailings management. Some examples include (but are not limited to) the following:

a. Water-based bitumen extraction methods are mature technologies and are key to current oil sands economics. They are unlikely to be replaced without significant development of new methods, with proven economics and commensurate (step change) benefits to tailings. In addition, they must also address environmental concerns. This is a major research initiative.

b. A 'position paper' that defines the current state of reclamation technologies, and casts a wide net in searching for 'new' technologies that have not been used in the oil sands industry to date, should be prepared.

4. The full potential of chemical amendments as technology enhancers has only begun to be addressed. Greater R\&D focus should be brought to bear on this issue, particularly by leveraging the knowledge of vendors who are active in industries outside of the oil sands, but who have not been tapped to date.

a. If industry expectation is that proponents take a particular tailings technology through Steps 1 to 3 (or 4 ) of the R\&D model prior to approaching oil sands operators, then the types of tests and standards for carrying them out should be clearly defined.

b. The OSTC should consider establishing or maintaining large laboratory and pilot scale facilities, which could be used by vendors or other third party proponents to demonstrate the effectiveness of their chemical amendment(s), after they have passed their 'entrance exam'.

c. The development of partnerships between oil sands industry operators and tailings technology providers should be further encouraged by establishing collaborative investments and testing programs, which would realise accelerated benefits from new technologies.

5. A more flexible regulatory approach that places more emphasis on achieving tailings reclamation and management objectives, and less emphasis on short-term goals, would result in more effective and less costly tailings reclamation. Government regulators should give serious consideration to updating current regulations. In doing so, consideration should be given to the following points:

a. Current regulations are focused on the shear strength of the soft tailings only, whereas trafficable surfaces require compatibility between the cap and the underlying soft tailings. Current plans and regulations for stabilisation, capping, and reclamation of soft tailings deposits should be re-examined for compatibility with material properties (both capping materials and underlying tailings), the scale of the operation, costs, and the desired landscape performance. Various methods for hydraulic capping should be included in this evaluation.

b. In a similar vein, current regulations assume particular capping and reclamation methods but exclude others due to restrictive criteria. Consideration should be given to technologies that are able to cap very soft deposits and promote early consolidation and strength gain, as opposed to the more traditional approaches that require access by mechanical equipment.

6. It became clear when carrying out the project work that issues of water balance and water chemistry are becoming critical and need attention. Initial efforts at defining water treatment needs were addressed in the project, but this issue is larger than just water treatment. A similar focus should be directed, in the future, at addressing water storage and water release issues as was given to tailings management issues herein.

The tailings roadmap project was a comprehensive compilation of information and opportunities in the present day oil sands industry. The final product of this project is a simple snapshot in time that looks at where we have come from and where we have the potential to go. At the time of this conference, the project report is already a year old. Industry has already mobilised to incorporate some of the technologies 
into their commercial practice, to advance research and development on others, and to continue to look for new technologies that can be added to and enhance tailings technology suites.

\section{Acknowledgements}

The authors of this paper would like to thank Rick Nelson from Alberta Innovates - Energy and Environment Solutions, and Alan Fair of the Oil Sands Tailings Consortium, for the permission to publish this paper. A thank you is extended to all the client representatives from the groups involved in the project, for all their contributions, words of encouragement, direction and comments made throughout the course of the project.

Heartfelt thanks are also given to the members of the Research Advisory Committee and the various project component leads.

A final thanks to Les Sawatsky for his contributions to the formation of the CTMC, and to Darcy Cowan, the project manager.

\section{References}

Alberta Innovates, Energy and Environment Solutions (2010) The technology deployment roadmap and action plan for "end-toend" solutions for oil sands tailings, Request for Proposal No. EES-10-02, Edmonton, Alberta, November 15, 2010.

Consortium of Tailings Management Consultants (2012a) Oil Sands tailings technology deployment roadmap project report Volume 1 project summary, viewed 28 August 2012, http://www.cosia.ca/projects/tailings.

Consortium of Tailings Management Consultants (2012b) Oil Sands tailings technology deployment roadmap project report Volume 2 C1 results, viewed 28 August 2012, http://www.cosia.ca/projects/tailings.

Consortium of Tailings Management Consultants (2012c) Oil Sands tailings technology deployment roadmaps project report Volume 3 C2 results, viewed 28 August 2012, http://www.cosia.ca/projects/tailings.

Consortium of Tailings Management Consultants (2012d) Oil Sands tailings technology deployment roadmaps project report Volume 4 C3 results, viewed 28 August 2012, http://www.cosia.ca/projects/tailings.

Consortium of Tailings Management Consultants (2012e) Oil Sands tailings technology deployment roadmap project report Volume 5 C4 results, viewed 28 August 2012, http://www.cosia.ca/projects/tailings.

Gidley, I. and Boswell, J. (2013) A generic model for technology development in oil sands tailings, in Proceedings 16th International Seminar on Paste and Thickened Tailings (Paste 2013), R.J. Jewell, A.B. Fourie, J. Caldwell and J. Pimenta (eds), 17-20 June 2013, Belo Horizonte, Brazil, Australian Centre for Geomechanics, pp. 549-560.

Sobkowicz, J.C. and Morgenstern, N.R. (2009) A Geotechnical Perspective on Oil Sands Tailings, in Proceedings of the 13th Annual Conference on Tailings and Mine Waste, Banff, Alberta, November 1-4. 
